\title{
Effectiveness and Safety of CGRP-mAbs in Menstrual- Related Migraine: A Real-World Experience
}

\author{
Marcello Silvestro - Ilaria Orologio • Simona Bonavita • \\ Fabrizio Scotto di Clemente · Carla Fasano • Alessandro Tessitore • \\ Gioacchino Tedeschi · Antonio Russo (D)
}

Received: April 12, 2021 / Accepted: May 13, 2021 / Published online: June 9, 2021

(C) The Author(s) 2021

\begin{abstract}
Introduction: Migraine shows a significantly higher prevalence in women, especially during reproductive age when menstrual-related hormonal fluctuations represent the most common migraine trigger. Indeed, over $50 \%$ of patients report a higher occurrence of migraine attacks during the perimenstrual window. Menstrual migraine attacks are consistently referred to as more disabling, less responsive to symptomatic treatments, longer in duration, and more prone to relapse than non-menstrual migraine attacks. Evidence strongly suggests that estrogen fluctuations are involved in migraine attacks worsening during the perimenstrual window through several mechanisms directly or indirectly involving the CGRP pathway. We aimed to evaluate whether mAbs blocking CGRP-
\end{abstract}

Marcello Silvestro and Ilaria Orologio contributed equally to this study.

Supplementary Information The online version contains supplementary material available at https:// doi.org/10.1007/s40122-021-00273-w.

M. Silvestro $\cdot$ I. Orologio $\cdot$ S. Bonavita

F. Scotto di Clemente - C. Fasano - A. Tessitore .

G. Tedeschi · A. Russo ( $\square)$

Department of Advanced Medical and Surgical Sciences (DAMS), Headache Center, University of Campania "Luigi Vanvitelli", Piazza Miraglia 2, 80138 Naples, Italy

e-mail: dottor.russo@gmail.com ligand or receptor (CGRP-mAbs) could represent an effective and safe preventive treatment for menstrual migraine attacks in patients with menstrual-related migraine (MRM) with previous treatment failures.

Methods: Forty patients with MRM with at least three previous treatment failures received monthly CGRP-mAbs. At the baseline and after six CGRP-mAbs administrations, patients underwent to extensive interviews to assess frequency, duration, intensity, and responsiveness to painkiller intake of migraine attacks occurring during the perimenstrual window.

Results: After six administrations of CGRPmAbs we observed a reduction of median menstrual migraine frequency (from 5 to 2 days per month), pain intensity (from $8 / 10$ to $6 / 10$ ), and attacks duration (from 24 to $8 \mathrm{~h}$ ) $(p<0.001)$. Nevertheless, a significant increase in the percentage of responding to migraine painkillers was observed from $42.5 \%$ at baseline to $95 \%$ at $\mathrm{T} 1(p<0.001)$.

Conclusions: CGRP-mAbs could represent a safe and effective preventive therapeutic strategy able to reduce the disabling burden of menstrual migraine attack frequency, duration, intensity, and significantly improve the response to painkillers. These findings could be related to and further indirectly prove the greater influence of CGRP-mediated mechanisms in the pathophysiology of menstrual migraine attacks. 
Keywords: Calcitonin gene-related peptide monoclonal antibody; Menstrual migraine; Migraine; Menstrual-related migraine

$\begin{array}{ll}\text { Abbreviations } \\ \text { CGRP } & \text { Calcitonin gene-related peptide } \\ \text { CGRP-mAbs } & \text { CGRP-monoclonal antibodies } \\ \text { MRM } & \text { Menstrual-related migraine } \\ \text { MM } & \text { Menstrual migraine } \\ \text { PMM } & \text { Pure menstrual migraine } \\ \text { CHD-3 } & \text { International Classification of } \\ & \text { Headache Disorders-3 } \\ \text { NSAIDs } & \text { Nonsteroidal anti-inflammatory } \\ & \text { drugs } \\ \text { COX2ibs } & \text { Cyclooxygenase 2 inhibitors } \\ \text { IHS } & \text { International Headache Society } \\ \text { MMD } & \text { Monthly migraine days } \\ \text { NRS } & \text { Numerical rating scale } \\ \text { AEs } & \text { Adverse events } \\ \text { SD } & \text { Standard deviation } \\ \text { IQR } & \text { Interquartile range } \\ \text { 5HT1R } & \text { 5-Hydroxytryptamine 1 receptor } \\ \text { PG } & \text { Prostaglandins } \\ \text { TRPV1 } & \text { Transient receptor potential } \\ & \text { vanilloid antagonist } \\ \text { MRI } & \text { Magnetic resonance imaging }\end{array}$

\section{Key Summary Points}

Menstrual migraine attacks are consistently referred to as more disabling, less responsive to symptomatic treatments, longer in duration, and more prone to relapse than non-menstrual migraine attacks.

Estrogen fluctuations are involved in migraine attacks worsening during the perimenstrual window through several mechanisms directly or indirectly involving the CGRP pathway.

CGRP-mAbs could represent a safe and effective preventive therapeutic strategy able to reduce menstrual migraine attack frequency, duration, and intensity, and significantly improve the response to painkillers.
Our findings further prove the greater influence of CGRP-mediated mechanisms in the pathophysiology of menstrual migraine attacks, supporting the role of CGRP-mAbs in menstrual migraine treatment.

\section{DIGITAL FEATURES}

This article is published with digital features, including a summary slide, to facilitate understanding of the article. To view digital features for this article go to https://doi.org/10.6084/ m9.figshare. 14579769

\section{INTRODUCTION}

Migraine is the most common neurological disorder and is actually the seventh-leading cause of disability worldwide [1] with an estimated prevalence of $12 \%$ in the general population [2]. Women have a 3.25 times greater risk of suffering from migraine attacks compared to men ( $18 \%$ of women vs. $6 \%$ of men), especially during reproductive age (until to $24 \%$ in those aged between 30 and 39 years) [3]. In this population, menstrual-related hormonal fluctuations represent the most common migraine trigger, found in over $50 \%$ of female patients reporting an increased migraine attack frequency and severity immediately before, during, or after menstruation [4,5]. This strong relationship led to the identification of menstrual migraine (MM), included in the appendix of the International Classification of Headache Disorders [6], where it is distinguished in (i) pure menstrual migraine (PMM) [ICHD-3 A1.1.1], affecting about $10 \%$ of females with migraine, with attacks limited to the perimenstrual window (that starts 2 days before the onset of menses and continues through the first 3 days of menstruation) and (ii) menstrual-related migraine (MRM) [ICHD-3 A1.1.2] with migraine attacks occurring both during the perimenstrual window and additionally at other times of the menstrual cycle.

Menstrual migraine attacks are consistently reported as more disabling, less responsive to 
symptomatic treatment, longer in duration, and more prone to relapse than non-menstrual migraine attacks [7]. Concerning MRM, although estrogenic supplementation therapy could be considered a therapeutic strategy, currently there are no specific anti-migraine drugs [8]. Short-term preventive treatments (from 2 days before to 5 days after the presumed beginning of menses) can be administered. On the other hand, these therapeutic strategies can be performed only in women with regular menstrual cycles [9] and with a not too high migraine attack frequency to avoid the risk of medication overuse development (since the most frequently used drugs are triptans, dihydroergotamine, NSAIDs, and COX2 inhibitors). Long-term daily prophylactic treatments can be equally administered in patients with MRM, but, although data supporting these strategies are low, they are believed to be only partially effective [10].

In the last years, mAbs blocking CGRP-ligand or receptor (CGRP-mAbs) have been developed. Data emerging from both randomized controlled trials and real-life experiences strongly supported CGRP-mAbs efficacy and tolerability as preventive treatments for both episodic and chronic migraine [11-14]. Interestingly, only in a post hoc subgroup analysis of a phase 3 , randomized, double-blind, placebo-controlled study of Erenumab ${ }^{\circledR}$ for prevention of episodic migraine the efficacy and safety of Erenumab in a subgroup of women with a history of MM has been supported [15].

However, to the best of our knowledge, no real-life experiences have specifically demonstrated the efficacy and safety of CGRP-mAbs to treat migraine attacks occurring during the perimenstrual window.

Therefore, we report the data emerging from our real-life experience using the CGRP-mAbs in patients with MRM and previous treatment failures, focusing on the perimenstrual window (e.g., from 2 days before to 3 days after the menses) and evaluating the attack frequency (migraine days), intensity, and duration as well as response to painkillers. Moreover, we evaluated the percentage of MRM patients experiencing $\geq 50 \%$ reduction of menstrual migraine days and attacks intensity. Finally, we show data on the CGRP-mAbs safety in these patients.

\section{METHODS}

\section{Study Design and Participants}

This is a report of our real-life experience focusing on the effectiveness and safety of mAbs anti-CGRP in the prevention and treatment of migraine attacks occurring during the perimenstrual window in patients with MRM with at least three or more previous preventive treatments.

Out of 93 migraine patients (of which 72 were female and 21 were male) from the migraine population being referred to the Headache Center of the Department of Neurology at the University of Campania "Luigi Vanvitelli" between February 2019 and January 2020, 42 menstruating women with migraine, according to the International Headache Society criteria (IHS), were identified. Among these, 40 patients fulfilling the IHS criteria for MRM and data from a 6-month follow-up were analyzed. All MRM patients had received and failed at least three or more oral preventive medication classes (beta-blockers, calcium-channel blockers, anticonvulsants, antidepressants, onabotulinumtoxinA) due to a lack of efficacy or intolerable side effects. Effectiveness failure was defined as $<50 \%$ reduction $(<30 \%$ in chronic migraine patients) in the frequency of migraine days after the administration of drugs for at least 3 months as recommended by the European Headache Federation treatment guidelines [16]. Tolerability failure was defined as documented discontinuation due to adverse events at any previous time of the treatment. Patients could take other preventive oral therapies with a stable dose for at least 3 months (6 months for patients who received onabotulinumtoxinA) before starting CGRP-mAbs.

Five patients received monthly Galcanezumab $^{\circledR}$, two patients received monthly Fremanezumab $^{\circledR}$, and 33 patients received, every 28 days, Erenumab $70 \mathrm{mg}$ (11 continued with monthly Erenumab $140 \mathrm{mg}$ after the third month). All patients were educated before 
starting the treatment to keep a headache diary to follow the progress of migraine attacks; it consisted of a table with the day of the month (up to 31) on the abscissa and the hours of the day (24) on the frames. The patient should mark the onset and the end of the attacks and the time of painkiller intake, allowing us the calculation of both the duration of the attacks and the response to the symptomatic drugs. In the case of headaches present at both the time of falling asleep and waking up, the night hours were included in the calculation of the attack. Among the additional information, the patient inserted the intensity of the attack (according to the NRS scale from 1 to 10) and the presence of symptoms associated with sensorineural hypersensitivity (photo/phono/osmophobia) and neurovegetative symptoms (nausea and vomiting). Finally, the patient entered in the appropriate boxes the days of menstruation, enabling to easily quantify the number and the days of menstrual migraine attacks.

At the first administration (T0) and after the six administration (T1) of CGRP-mAbs, all patients underwent an extensive interview to examine clinical parameters of disease severity such as migraine days per month (MMD), menstrual migraine days, menstrual migraine average pain intensity (assessed by numerical rating scale [NRS]), menstrual migraine attacks duration, and response to painkillers. Moreover, the percentage of MRM patients experiencing $\geq 50 \%$ reduction of menstrual migraine days and attack duration was evaluated. During the period of observation, all adverse events (AEs) related to the drug were recorded and used as a safety measure. Each patient gave informed consent for the analysis and publication of the data. The study was conducted in accordance with the International Council for Harmonization guidelines and the study protocol was approved by the local Ethics Board of University of Campania "Luigi Vanvitelli" (code 30,564/ 20).

\section{Outcome Measures}

We focused on migraine attacks occurring during the perimenstrual window (from 2 days prior to the first 3 days of menstruation) in patients with MRM evaluating the CGRP-mAbs effects on their frequency, pain severity, duration, and responsiveness to painkillers. The response to painkillers was evaluated at $2 \mathrm{~h}$ after intake; the patients were instructed not to repeat the painkiller assumption in the $2 \mathrm{~h}$ after the first intake. If patients repeated the painkiller intake or the headache persisted after $2 \mathrm{~h}$, the attack was classified as non-responsive to rescue therapy. Moreover, the rescue therapy usually used by the patients was not changed throughout the observation period. Besides, we consider the effectiveness of CGRP-mAbs treatment on the total MMD (e.g. $\geq 50 \%$ reduction from the baseline of MMD for episodic migraine and $\geq 30 \%$ for chronic migraine). Effectiveness was evaluated at the end of the observation period through detailed interviews and analysis of headache diaries. Finally, we performed a post hoc analysis considering 21 patients treated only with CGRP-mAbs monotherapy.

Safety and tolerability were assessed by recording self-reported and observed adverse events by physical examination.

\section{Statistical Analysis}

All demographic and clinical data were checked for normality using Shapiro-Wilk test. Continuous variables are reported as mean \pm standard deviation (SD), categorical variables are expressed as median \pm interquartile range, rates values are reported as subject counts and percentage. Since data did not conform to normal distribution, the Wilcoxon rank test was used to compare median MMD, pain intensity, monthly menstrual migraine days, and migraine attack duration. Finally, the McNemar's test was used to compare the response to painkillers. Due to the observational character 
of the study, the statistical power has not been performed. Statistical significance was set at $p<0.05$. All analyses were performed using SPSS version-14 (StataCorp, College Station, TX, USA).

\section{RESULTS}

\section{Demographic Characteristics}

The whole population consisted of 40 patients fulfilling the ICHD-3 criteria for MRM [ICHD-3 A 1.1.2]. The mean age was $39.35 \pm 9.17$ years (range, 18-51) and the mean age of onset of migraine in our population was $15.52 \pm 7.61$ years. The median frequency of menstrual attacks at baseline (T0) was 5 (IQR 1.25), while the median MMD was 19 (IQR 10). The median duration of menstrual attacks was $24 \mathrm{~h}$ (IQR 42), the median intensity (evaluated by NRS score) was 8 (IQR 1.25), and the percentage of responders to painkillers (defined as "pain-free at $2 \mathrm{~h}$ " after intake of symptomatic drugs) was $42.5 \%$ (Table 1).

\section{Effectiveness}

After six CGRP-mAbs administrations, we observed a statistically significant improvement in frequency, duration, average pain severity, and responsiveness to painkillers of migraine attacks occurring during the perimenstrual window $(p<0.001)$ as well as MMD. More specifically, our population showed a statistically significant improvement in the frequency of menstrual migraine, from baseline median frequency of 5 (IQR: 1.25) to T1 median frequency of 2 days (IQR: 2$)$ per month $(p<0.001)$ (Fig. 1). Moreover, after six CGRP-mAbs administrations, the duration of menstrual migraine attacks was reduced from a baseline median duration of $24 \mathrm{~h}$ (IQR: 42 ) to $8 \mathrm{~h}$ (IQR: 20) at $\mathrm{T} 1$, while headache pain severity improved from a median pain intensity score (evaluated by NRS) of 8 (severe pain; IQR: 1.25 ), to 6 (moderate pain; IQR: 2.25$) \quad(p<0.001)$ (Fig. 2). Nevertheless, a significant increase in the percentage of patients responding to migraine painkiller was observed from $42.5 \%$ at baseline to $95 \%$ at T1 $(p<0.001)$ (Fig. 3). Finally, MMD improved from T0 median frequency of 19 (IQR: 10) to T1 median frequency of 5 days (IQR: 6) $(p<0.001)$. Furthermore, $57.5 \%$ (23) of the patients reported a $\geq 50 \%$ reduction of menstrual migraine attacks, $62.5 \%$ (25) of patients experienced $a \geq 50 \%$ reduction menstrual migraine attack duration and, finally, $62.5 \%$ (25 pts) switched from a severe migraine attacks pain intensity ( $\geq 8$ according NRS score) to a moderate pain intensity $(<8$ according NRS score). CGRP-mAbs therapy was (i) effective in

Table 1 Baseline demographic and clinical parameters $(N=40)$

\begin{tabular}{ll}
\hline Age $($ mean \pm SD) & $\mathbf{3 9 . 4 \pm 9 . 1 7}$ \\
\hline Age at migraine onset (mean \pm SD) & $15.52 \pm 7.61$ \\
Disease history (mean years \pm SD) & $26.24 \pm 10.07$ \\
MMD (median $\pm \mathrm{IQR})$ & $19 \pm 10$ \\
Menstrual migraine attacks (median $\pm \mathrm{IQR})$ & $5 \pm 1.25$ \\
Previous preventive medication classes failure & $4.3 \pm 0.8$ \\
Responders to painkiller ${ }^{a}$ of menstrual migraine $[n(\%)]$ & $42.5 \%$ \\
Menstrual migraine duration (hours $\pm \mathrm{IQR})$ & $24 \pm 42$ \\
Menstrual migraine pain intensity (NRS $\pm \mathrm{IQR})$ & $8 \pm 1.25$ \\
\hline
\end{tabular}

$S D$ standard deviation, $M M D$ monthly migraine days, $I Q R$ interquartile range, $N R S$ numerical rating scale $(0-10)$

${ }^{a}$ Pain-free at $2 \mathrm{~h}$ after painkiller intake 


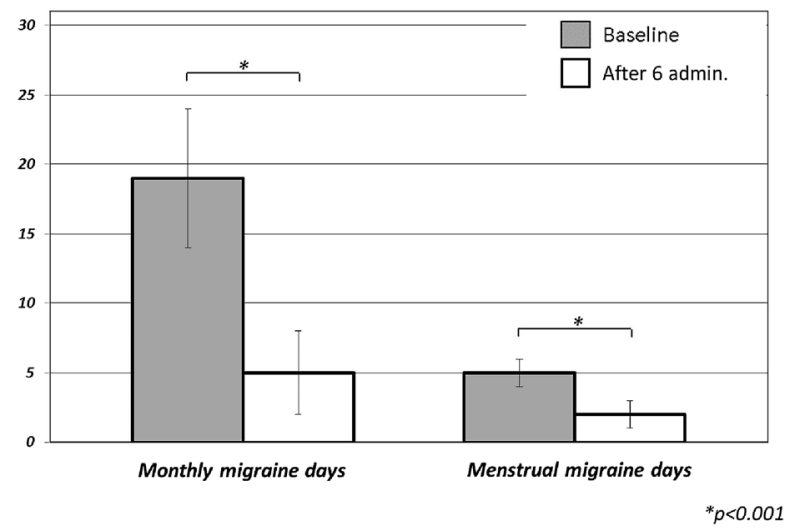

Fig. 1 Comparison of menstrual migraine days per month and monthly migraine days (MMD) at baseline (T0) and after six administrations of CGRP-mAbs (T1)

31 (77.5\%) patients for both menstrual migraine attacks (occurring in perimenstrual window) and MMD; (ii) effective in two patients only in MMD but not of menstrual migraine attacks (5\%); (iii) effective in two patients (5\%) only in menstrual attacks. In five patients $(12.5 \%)$, CGRP-mAbs therapy was ineffective in both menstrual migraine attacks and MMD (Table 2). Finally, in a post hoc analysis we considered 21 patients who were receiving only CGRP-mAbs, without other preventive treatments, and we found an equally statistically significant reduction in the frequency, intensity, and duration of menstrual migraine episodes. Specifically, the frequency of menstrual migraine changed from baseline (T0) median

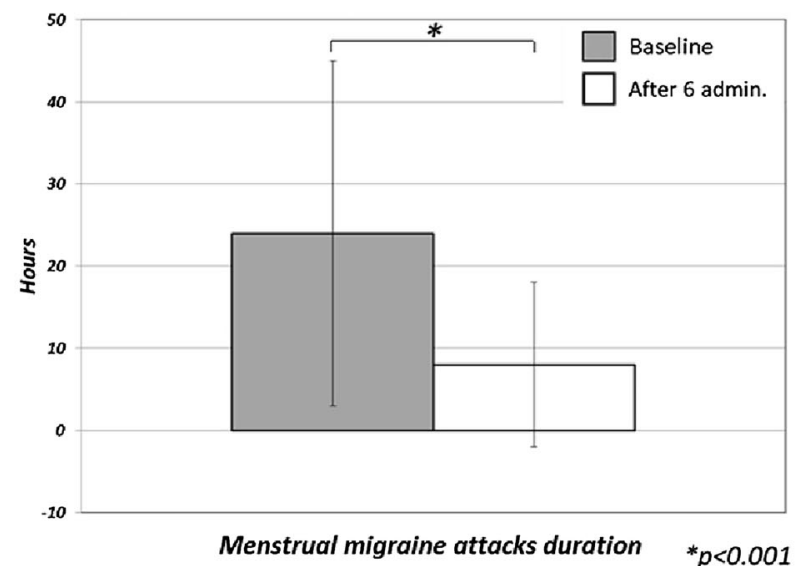

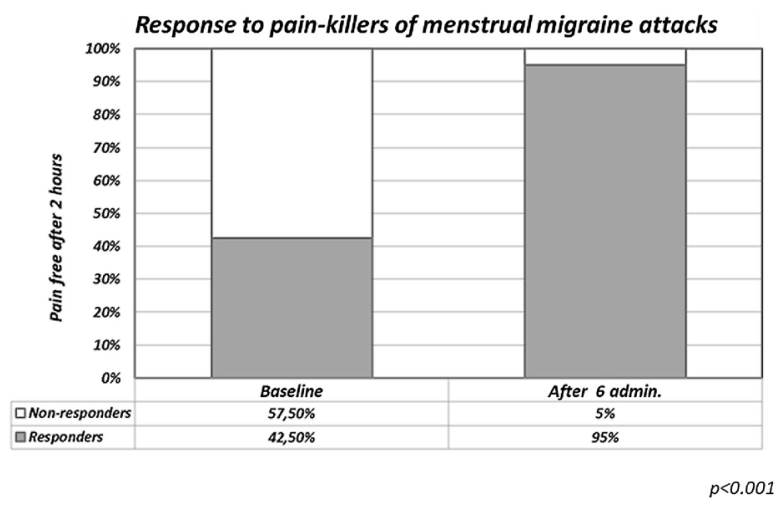

Fig. 3 Responsiveness to painkillers (defined as pain-free at $2 \mathrm{~h}$ after painkiller intake) at baseline (T0) and after six administrations of CGRP-mAbs (T1)

frequency of five migraine days per month (IQR: 1) to T1 median frequency of 2 days per month (IQR: 1) $(p<0.001)$; the duration of menstrual migraine attacks reduced from a baseline (T0) median duration of $24 \mathrm{~h}$ (IQR: 24) to $\mathrm{T} 1$ median duration of $7 \mathrm{~h}$ (IQR: 11.25); finally, the headache severity improved from a baseline (T0) median intensity (evaluated by NRS) of $8 / 10$ (severe pain; IQR: 1 ), to T1 median intensity of 6/10 (moderate pain; IQR: 1.5) $(p<0.001)$.

\section{Safety and Tolerability}

During observation (from T0 to T1), we recorded adverse events (AE) in $22.5 \%$ of patients:

Fig. 2 Comparison of duration (hours) and intensity of pain (NRS scores) at baseline (T0) and after six administrations of CGRP-mAbs (T1)

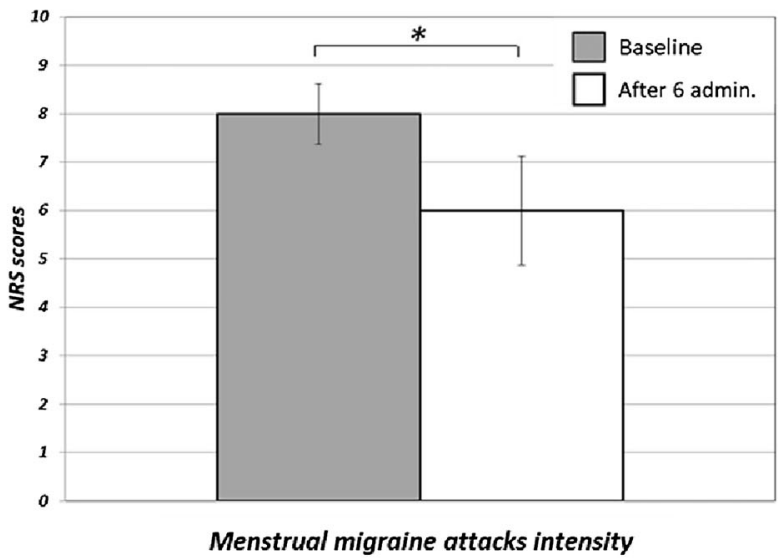


Table 2 Effectiveness of CGRP-mAbs after six administrations

\begin{tabular}{|c|c|c|c|}
\hline Outcome & Baseline & $\begin{array}{l}\text { Sixth } \\
\text { administration }\end{array}$ & $p$ value \\
\hline Menstrual migraine days (median $\mathrm{MM} \pm \mathrm{IQR}$ ) & $5 \pm 1.25$ & $2 \pm 2$ & $<0.001$ \\
\hline Menstrual migraine duration (median hours $\pm \mathrm{IQR}$ ) & $24 \pm 42$ & $8 \pm 20$ & $<0.001$ \\
\hline Menstrual migraine pain intensity (Median NRS \pm IQR) & $8 \pm 1.25$ & $6 \pm 2.25$ & $<0.001$ \\
\hline Menstrual migraine responders to painkillers ${ }^{\mathrm{a}}[n(\%)]$ & $42.5 \%$ & $95 \%$ & $<0.001$ \\
\hline MMD (median MMD $\pm \mathrm{IQR})$ & $19 \pm 10$ & $5 \pm 6$ & $<0.001$ \\
\hline$\%$ of pts with $>50 \%$ reduction in menstrual migraine days & & $57.5 \%$ (23 patients) & \\
\hline$\%$ of patients with $>50 \%$ reduction in menstrual migraine duration & & $62.5 \%$ (25 patients) & \\
\hline$\%$ of patients switching from severe to moderate pain intensity & & $62.5 \%$ (25 patients) & \\
\hline $\begin{array}{l}\% \text { of patients with significant reduction in } \mathrm{MMD}^{\mathrm{b}} \text { and menstrual migraine } \\
\text { days }\end{array}$ & & $77.5 \%$ (31 patients) & \\
\hline$\%$ of patients with significant reduction only in MMD & - & $5 \%$ (two patients) & \\
\hline$\%$ of patients with significant reduction only in menstrual migraine days & - & $5 \%$ (two patients) & \\
\hline$\%$ of patients with not significant reduction in MMD and menstrual migraine & - & $12.5 \%$ (five patients) & \\
\hline
\end{tabular}

$I Q R$ interquartile range, $M M D$ monthly migraine days, $N R S$ numerical rating scale

a Pain-free at $2 \mathrm{~h}$ after painkiller intake

b $\geq 30 \%$ in chronic migraine patients; $\geq 50 \%$ in episodic migraine patients

constipation (20\%), fatigue $(2.5 \%)$, and nausea $(2.5 \%)$ were the most frequently reported during treatment. There were no serious AEs and no patient discontinued treatment due to adverse events.

\section{DISCUSSION}

In the present real-life experience, we observed a significant reduction in the frequency, duration, and intensity of migraine attacks occurring during the perimenstrual window as well as in the response to painkiller in a cohort of MRM patients, with previous treatment failures, during the therapy with CGRP-mAbs for a period of 6 months.

Migraine represents the most common neurologic disorder with a significantly higher prevalence in women witnessed by a sex ratio of about 3:1 [17]. Over 50\% of female migraine patients report a strong relationship between menstruation and migraine $[4,5]$, characterized by the higher occurrence of migraine attacks during the perimenstrual window (which starts 2 days before the onset of menses and continues through the first 3 days of menstruation). Indeed, menstrual-related hormonal fluctuations have been identified as the most common migraine trigger during the reproductive age. This relationship leads to define the so-called $\mathrm{MM}$ and, according to the ICHD-3 criteria, its two declensions of PMM (A. 1.1.1), where the attacks are strictly confined to the perimenstrual window, and MRM (A. 1.1.2), characterized by attacks occurring both during the perimenstrual window and at other times of the menstrual cycle [6]. It is noteworthy that migraine attacks occurring during the perimenstrual window are characterized by higher pain duration and intensity with poor response to acute medications when compared to migraine attacks occurring outside menstruation [7]. The mechanisms underlying MM have 
been widely investigated. There is a functional interaction between ovarian steroid hormones, CGRP and the trigeminal vascular system [18], where the presence of sex hormone receptors suggests a sensibility to variations in the levels of these hormones [19].

The fall of estrogen levels (observed immediately before menstruation) has been identified as the possible cause of MM [20]. Indeed, a higher baseline estrogen level and a consequent greater estrogenic withdrawal during the luteal phase are associated with increased prevalence of menstrual migraine attacks, with an inverse relationship between migraine severity and urinary estrogen levels during the perimenstrual window [21]. When estrogen levels fall, production of serotonin becomes reduced, an increase of monoamine oxidase activity causes the acceleration of serotonin elimination rate, and the sensitivity of 5-hydroxytryptamine1 (5HT1) receptors (triptans target) decreases [21]; this could explain the reduced response to triptans in menstrual migraine [22]. Moreover, estrogen fall may increase the susceptibility to prostaglandins (PG), as witnessed by the three-fold increase in PG levels observed between the follicular and luteal phases, and even more during menstruation [23, 24]. PG can cause neurogenic inflammation by promoting the release of CGRP neuropeptides substance $\mathrm{P}$ and neurokinins, thereby increasing the susceptibility of blood vessels to PG, which has been implicated in neurogenic inflammation [24, 25].

Furthermore, changes in estrogen concentration during menstruation influences the sensitivity of the transient receptor potential vanilloid antagonist (TRPV1) receptors, therefore increasing the amount of CGRP in the terminals of perivascular nerves [26].

Altogether, these findings suggest that estrogen fluctuations are involved in migraine attacks worsening during the perimenstrual window through several mechanisms directly or indirectly involving the CGRP pathway [26].

Moreover, the abnormal brain responses to thermal pain stressor during interictal period using a used high-field magnetic resonance imaging (MRI) have been evaluated in male and female migraine patients [27]. A stronger activation of the spinal trigeminal nucleus in female migraine patients compared to male migraine patients and healthy controls was observed and the differences in sensitivity of the trigeminal system attributed to hormonal influences [27], shedding light on the gender differences in migraine pathophysiology.

To date, many studies have been performed to evaluate the effectiveness of antimigraine acute treatments, short-term prophylaxis, and daily preventive approaches in patients with MM. However, hormone supplementation therapy seems to be effective in preventing MM attacks by means of estrogen level stabilization since fluctuations, especially sudden drops, can precipitate migraine attacks [28]. Long half-life triptans, NSAIDs, COX2 ibs, magnesium, and estrogen supplementation have been suggested as preventive therapies during the time of highest risk of menstrual migraine attacks in PMM patients with regular menses, to reduce the burden associated with long-term daily preventive medications [9]. Several hormonal therapeutic approaches have been evaluated: percutaneous gel formulation of estradiol (from day -2 to day +5 of cycle, or daily from the 10th day following the first day of peak fertility and to day +2 of cycle), patch administration of estradiol (from day -4 to day +4 of cycle) as well as continuous hormonal contraception (estrogen or progesterone or combination of these) [10]. Estrogen supplementation appears to be effective in reducing the number of headache days also in MRM, although several studies reported an increase in headache days when supplementation is discontinued [29]. Collectively, these studies have shown mixed results as migraine preventive treatment and should be used with caution after a careful risk to benefit evaluation, in particular on cerebrovascular ischemic risk factors [10, 28].

Contrariwise, daily preventive treatments are ideal for MRM [30], but data about the best long-term prophylactic strategy for these patients are poor and standard migraine prophylactic agents are currently the pivot of therapy [10]. In this frame, topiramate as preventive treatment in women with MRM has been shown to be effective in reducing menstrual migraine attack frequency, but not severity or duration [31]. 
Indeed, patients with high-frequency episodic migraine or chronic migraine, associated with severe menstrual migraine attacks, shortterm preventive therapies with NSAIDs and/or triptans, could induce or worsen a medication overuse, increasing the risk of migraine chronification [6]. Nonetheless, only about 30\% of patients benefit from these therapeutic approaches [22, 32].

Since migraine patients belonging to our sample had previously failed at least three longterm daily preventive treatments and considering the involvement of the CGRP pathway in the mechanisms by which hormonal changes impact on the migraine attacks during menstruation, we explored the effectiveness and safety of CGRPmAbs in MRM. Interestingly, a statistically significant reduction in frequency of menstrual migraine days (from 5 days at baseline to 2 days), duration of attacks (from 24 to $8 \mathrm{~h}$ ), pain intensity (from a NRS median of 8/10-6/10) and response to painkiller (from 42.5 to $95 \%$ ) were demonstrated after six CGRP-mAbs administrations. Interestingly, the reduction in non-menstrual migraine episodes experienced by the patients appeared slightly higher in percentage than that in menstrual migraine episodes (from 14.5 to $4,72.4 \%$ vs. from 5 to $2,60 \%$ ). This finding could further strengthen the notion that menstrual migraine is more resistant and less responsive to prophylactic drug therapies. Furthermore, there were no patient-reported serious AEs or decisions to discontinue the CGRP-mAbs treatment due to poor tolerability.

In line with our observation, it has been very recently demonstrated that $\geq 2$ acute medications per day were higher during menstrual than in non-menstrual headache days in erenumab non-responders but not in erenumab responders. These findings indirectly suggest an overall reduced attack duration or increased painkiller responsiveness during menstrual days in erenumab responders [33]. Furthermore, our findings fit well with those emerging from a post hoc analysis of the STRIVE RCT trial (a phase 3, randomized, double-blind, placebo-controlled study of Erenumab for prevention of episodic migraine) [15]. However, these data, collected in the context of a prospective clinical trial, were not focused on migraine attacks occurring during the perimenstrual window but supported the efficacy of Erenumab on the monthly migraine attacks in women with self-reported menstrual migraine attacks. Our study firstly described the effect of CGRP-mAbs on MRM in a uniform sample of menstruating women with episodic high-frequency migraine or chronic migraine, who had previously failed at least three antimigraine preventive therapies. The data emerging from the present real-life experience show that mAbsCGRPs are equally effective in treating menstrual and non-menstrual migraine attacks differently from what previously observed with other preventive migraine treatment. This finding could be related to and further indirectly prove the greater influence of CGRP-mediated mechanisms in the pathophysiology of menstrual migraine attacks.

However, we are aware that our study is not exempt from some limitations. First of all, being a non-randomized open-label study, there was no placebo or active comparator arm as is usual in the real-word experiences. However, an openlabel design is informative when the efficacy and safety profile of treatment is well established, as it is with CGRP-mAbs for episodic and chronic migraine. Moreover, a comparative evaluation between the three $\mathrm{mAbs}$ anti-CGRP administered was not possible due to the small number of patients treated with Galcanezumab (12.5\% of the sample under study $=5$ women) and Fremanezumab $(5 \%=2$ women $)$ compared to patients treated with Erenumab $(82.5 \%=33$ women). Finally, since the CGRP-mAbs treatment was added to other preventive medications, to further evaluate the efficacy of CGRPmAbs as not dependent from synergistic or additive effect with other concomitant preventive therapies, we performed a post hoc analysis considering patients treated with CGRP-mAbs monotherapy. Despite the low sample size, we keep finding a statistically significant reduction in the frequency, intensity, and duration of menstrual migraine attacks.

\section{CONCLUSIONS}

The present findings demonstrate that mAbs anti-CGRP ligand (Fremanezumab and Galcanezumab) or receptor (Erenumab) could 
represent an effective and safe therapeutic strategy for menstrual migraine attacks, significantly improving the frequency, duration, intensity, and response to painkillers. Further studies with a larger sample size and a longer period of observation are needed to better clarify these preliminary findings as well as to demonstrate the effectiveness and safety of mAbs CGRP also in PMM patients.

\section{ACKNOWLEDGEMENTS}

Funding. This research received no specific grant from any funding agency in the public, commercial, or not-for-profit sectors. The journal's Rapid Service Fee was funded by the authors.

Authorship. All named authors meet the International Committee of Medical Journal Editors (ICMJE) criteria for authorship for this article, take responsibility for the integrity of the work as a whole, and have given their approval for this version to be published.

Authorship Contributions. Marcello Silvestro: data analysis, literature review, results interpretation, manuscript drafting and revision; Ilaria Orologio: data analysis, literature review, results interpretation, manuscript drafting and revision; Simona Bonavita: results interpretation, manuscript revision; Fabrizio Scotto di Clemente literature review, results interpretation, manuscript revision; Carla Fasano: results interpretation, manuscript revision; Alessandro Tessitore: data analysis, results interpretation, manuscript revision; Gioacchino Tedeschi: results interpretation, manuscript revision; Antonio Russo: data analysis, results interpretation, manuscript drafting and revision.

Disclosures. The author(s) declare the following potential conflicts of interest with respect to the research, authorship, and/or publication of this article: Marcello Silvestro has received speaker honoraria from Novartis, Lilly, and Teva. Alessandro Tessitore has received speaker honoraria from Novartis, Schwarz Pharma/UCB, Lundbeck, AbbVie, and Glaxo. Gioacchino Tedeschi has received speaker honoraria from Sanofi-Aventis, Merck Serono, Bayer Schering Pharma, Novartis, Biogen-Dompé AG, Teva and Lilly; has received funding for travel from Bayer Schering Pharma, Biogen-Dompé AG, Merck Serono, Novartis, and Sanofi Aventis; and serves as an associate editor of Neurological Sciences. Antonio Russo has received speaker honoraria from Allergan, Lilly, Novartis, and Teva and serves as an associate editor of Frontiers in Neurology (Headache Medicine and Facial Pain session). Ilaria Orologio, Simona Bonavita, Fabrizio Scotto di Clemente, and Carla Fasano have nothing to declare.

Compliance with Ethics Guidelines. The study has been conducted in accordance with the International Council for Harmonization guidelines and the study protocol was approved by the local Ethics Board of University of Campania "Luigi Vanvitelli" (code 30,564/20). Each patient provided informed consent for the analysis and publication of the data.

Data Availability. All data are available and can be requested at the following e-mail: dottor.russo@gmail.com.

Open Access. This article is licensed under a Creative Commons Attribution-NonCommercial 4.0 International License, which permits any non-commercial use, sharing, adaptation, distribution and reproduction in any medium or format, as long as you give appropriate credit to the original author(s) and the source, provide a link to the Creative Commons licence, and indicate if changes were made. The images or other third party material in this article are included in the article's Creative Commons licence, unless indicated otherwise in a credit line to the material. If material is not included in the article's Creative Commons licence and your intended use is not permitted by statutory regulation or exceeds the permitted use, you will need to obtain permission directly from the copyright holder. To view a copy of this licence, visit http://creative commons.org/licenses/by-nc/4.0/. 


\section{REFERENCES}

1. Vetvik KG, MacGregor EA. Sex differences in the epidemiology, clinical features, and pathophysiology of migraine. Lancet Neurol. 2017;16(1):76-87.

2. Yeh WZ, Blizzard L, Taylor BV. What is the actual prevalence of migraine? Brain Behav. 2018;8(6): e00950.

3. Allais G, Chiarle G, Sinigaglia S, Airola G, Schiapparelli $\mathrm{P}$, Benedetto C. Gender-related differences in migraine. Neurol Sci. 2020;41(Suppl 2):429-36.

4. Mannix LK, Calhoun AH. Menstrual Migraine. Curr Treat Options Neurol. 2004;6(6):489-98.

5. Allais G, Chiarle G, Sinigaglia S, et al. Treating migraine with contraceptives. Neurol Sci. 2017;38(Suppl 1):85-9.

6. Headache Classification Committee of the International Headache Society (IHS) The International Classification of Headache Disorders, 3rd edition. Cephalalgia. 2018;38(1):1-211.

7. $\mathrm{Hu} \mathrm{Y,} \mathrm{Guan} \mathrm{X,} \mathrm{Fan} \mathrm{L,} \mathrm{Jin} \mathrm{L.} \mathrm{Triptans} \mathrm{in} \mathrm{prevention}$ of menstrual migraine: a systematic review with meta-analysis. J Headache Pain. 2013;14(1):7 (Published 2013 Jan 30).

8. MacGregor EA. Prevention and treatment of menstrual migraine. Drugs. 2010;70(14):1799-818.

9. Allais G, Chiarle G, Sinigaglia S, Benedetto C. Menstrual migraine: a review of current and developing pharmacotherapies for women. Expert Opin Pharmacother. 2018;19(2):123-36.

10. Silberstein SD, Hutchinson SL. Diagnosis and treatment of the menstrual migraine patient. Headache. 2008;48(Suppl 3):S115-23.

11. Lattanzi S, Brigo F, Trinka E, et al. Erenumab for preventive treatment of migraine: a systematic review and meta-analysis of efficacy and safety. Drugs. 2019;79(4):417-31.

12. Lionetto L, Curto M, Cisale GY, et al. Fremanezumab for the preventive treatment of migraine in adults. Expert Rev Clin Pharmacol. 2019;12(8):741-8.

13. Detke HC, Goadsby PJ, Wang S, Friedman DI, Selzler KJ, Aurora SK. Galcanezumab in chronic migraine: the randomized, double-blind, placebocontrolled REGAIN study. Neurology. 2018;91(24): e2211-21.

14. Stauffer VL, Dodick DW, Zhang Q, Carter JN, Ailani J, Conley RR. Evaluation of galcanezumab for the prevention of episodic migraine: the EVOLVE-1 Randomized Clinical Trial. JAMA Neurol. 2018;75(9):1080 (1088 published correction appears in JAMA Neurol. 2019 Jul 1;76(7):872).

15. Pavlovic JM, Paemeleire K, Göbel H, et al. Efficacy and safety of erenumab in women with a history of menstrual migraine. J Headache Pain. 2020;21(1): 95 (Published 2020 Aug 3).

16. Sacco S, Bendtsen L, Ashina M, et al. European headache federation guideline on the use of monoclonal antibodies acting on the calcitonin gene related peptide or its receptor for migraine prevention. J Headache Pain. 2019;20(1):6 (2019 May 23;20(1):58 Published 2019 Jan 16).

17. Burch R, Rizzoli P, Loder E. The Prevalence and Impact of Migraine and Severe Headache in the United States: figures and Trends From Government Health Studies. Headache. 2018;58(4): 496-505.

18. Labastida-Ramírez A, Rubio-Beltrán E, Villalón CM, MaassenVanDenBrink A. Gender aspects of CGRP in migraine. Cephalalgia. 2019;39(3):435-44.

19. Gupta S, McCarson KE, Welch KM, Berman NE. Mechanisms of pain modulation by sex hormones in migraine. Headache. 2011;51(6):905-22.

20. Calhoun $\mathrm{AH}$. Understanding menstrual migraine. Headache. 2018;58(4):626-30.

21. MacGregor EA, Frith A, Ellis J, Aspinall L, Hackshaw A. Incidence of migraine relative to menstrual cycle phases of rising and falling estrogen. Neurology. 2006;67(12):2154-8.

22. Maasumi K, Tepper SJ, Kriegler JS. Menstrual migraine and treatment options: review. Headache. 2017;57(2):194-208.

23. Downie J, Poyser NL, Wunderlich M. Levels of prostaglandins in human endometrium during the normal menstrual cycle. J Physiol. 1974;236(2): 465-72.

24. Cupini LM, Corbelli I, Sarchelli P. Menstrual migraine: what it is and does it matter? J Neurol. 2020. https://doi.org/10.1007/s00415-020-09726-2 (published online ahead of print, 2020 Jan 28).

25. Matsuda M, Huh Y, Ji RR. Roles of inflammation, neurogenic inflammation, and neuroinflammation in pain. J Anesth. 2019;33(1):131-9.

26. Ibrahimi $\mathrm{K}$, Danser $\mathrm{AHJ}$, Villalón $\mathrm{CM}$, van den Meiracker $\mathrm{AH}$, MaassenVanDenBrink A. Influence of varying estrogen levels on trigeminal CGRP release in healthy women. J Headache Pain. 2013;14(Suppl 1):P123. 
27. Maleki N, Linnman C, Brawn J, Burstein R, Becerra L, Borsook D. Her versus his migraine: multiple sex differences in brain function and structure. Brain. 2012;135(Pt 8):2546-59.

28. Mathew PG, Dun EC, Luo JJ. A cyclic pain: the pathophysiology and treatment of menstrual migraine. Obstet Gynecol Surv. 2013;68(2):130-40.

29. Sullivan E, Bushnell C. Management of menstrual migraine: a review of current abortive and prophylactic therapies. Curr Pain Headache Rep. 2010;14(5):376-84.

30. Silberstein S, Patel S. Menstrual migraine: an updated review on hormonal causes, prophylaxis and treatment. Expert Opin Pharmacother. 2014;15(14):2063-70.
31. Allais G, Sanchez del Rio M, Diener HC, et al. Perimenstrual migraines and their response to preventive therapy with topiramate. Cephalalgia. 2011;31(2):152-60.

32. Ferrari MD, Goadsby PJ, Roon KI, Lipton RB. Triptans (serotonin, 5-HT1B/1D agonists) in migraine: detailed results and methods of a meta-analysis of 53 trials. Cephalalgia. 2002;22(8):633-58 (published correction appears in Cephalalgia. 2003 Feb;23(1):71.).

33. Ornello R, Frattale I, Caponnetto V, De Matteis E, Pistoia F, Sacco S. Menstrual headache in women with chronic migraine treated with erenumab: an observational case series. Brain Sci. 2021;11(3):370. 